Relations industrielles

Industrial Relations

\title{
Wage Incentive Systems, par Garth L. Mangum - Institute of Industrial Relations, University of California, Berkeley, 1964, 83 pages.
}

\section{Jean-Paul Deschênes}

Volume 19, numéro 4, 1964

URI : https://id.erudit.org/iderudit/027539ar

DOI : https://doi.org/10.7202/027539ar

Aller au sommaire du numéro

Éditeur(s)

Département des relations industrielles de l'Université Laval

ISSN

0034-379X (imprimé)

1703-8138 (numérique)

Découvrir la revue

Citer ce compte rendu

Deschênes, J.-P. (1964). Compte rendu de [Wage Incentive Systems, par Garth L. Mangum - Institute of Industrial Relations, University of California, Berkeley, 1964, 83 pages.] Relations industrielles / Industrial Relations, 19(4), 532-532. https://doi.org/10.7202/027539ar

Tous droits réservés (C Département des relations industrielles de l'Université Laval, 1964
Ce document est protégé par la loi sur le droit d'auteur. L'utilisation des services d'Érudit (y compris la reproduction) est assujettie à sa politique d'utilisation que vous pouvez consulter en ligne.

https://apropos.erudit.org/fr/usagers/politique-dutilisation/ 
Wage Incentive Systems, par Garth L Mangum - Institute of Industrial Relations, University of Colifornio, Berkeley, 1964, 83 poges.

Si le rythme de publication dans un domaine particulier est un signe de popularité, l'utilisation des systèmes de rémunération au rendement vo en diminuant. En effet, duront les cina dernières années on a peu écrit sur le sujet et le modeste volume de monsieur Mangum est à signaler.

La première portie du volume est consacrée à la description sommaire de quelques systèmes de rémunération au rendement, individuels et collectifs. II s'ogit d'un exposé de faits sans tentation d'évaluation ou d'appréciation.

Le reste du volume s'ottoche aussi ò décrire des situotions sans vouloir les analyser ou les interpréter. Voici quelques observations dont l'outeur nous fait part:

1 - Les systèmes de rémunérotion au rendement étoblissent une relation plus ou moins directe entre les gages et le niveau du rendement.

2-On ne peut signaler aucune tendance quant à l'utilisotion de tels systèmes. Des compagnies adoptent une rémunération au rendement, d'autres l'abandonnent.

3-En général les gains basés sur le rendement sont de 15 à 35 pour cent au dessus des toux horaires.

4-II n'existe pos d'attitude uniforme des unions à l'égard des systèmes. Les politiques diffèrent d'une union à l'autre, à l'intérieur des unions, et d'une période à l'autre.
5- La rémunération au rendement tend ò s'appliquer aux fonctions non mesurables comme les fonctions reiiées ò l'entretien.

6-De plus en plus le rythme de la production est conditionné par la machinerie et dans ces circonstances, l'application de la rémunération ou rendement soulève plusieurs questions.

7-ll existe une tendance ò rendre plus làche les temps normalisés, ce qui entraine une modification de la structure des salaires, des coûts de la main-d'oeuvre plus élevés et des temps inexacts considérés comme normaux.

8-On accepte généralement le droit de la direction de reviser les temps normalisés a la suite de changements dans l'équipement, le matériel et le processus de fabrication.

9-Un toux horaire minimum est presque toujours goranti.

10-On protège aussi les gages des travailleurs dans les cas d'arrêt de procuction, de temps normalisés inexacts et de conditions difficiles.

11 -Les employés sont assurés d'aucune diminution de leurs gains lorsque les temps normalisés sont revisés.

II s'agit d'un volume qui peut intéresser le personnel de côdre ou les unions ouvrières oux prises ovec le problème de la rémunération au rendement.

Jean-Poul Deschênes

\section{PUBLICATIONS RECENTES RECENT PUBLICATIONS}

\section{Généralités}

«Lobour and the Scientic Revolution 》, by Harold Wilson, Information, United Steel. workers of Americo, Vol. 12, no 2, April 1964, pp. 1-7.
«How to End Poverty », by Dean Russell, The Freeman, Vol. 14 no 8, August 1964, pp. $3-13$.

«Effective Learning and Cooperative Education », by Dr Clarence Leuba, A poper de- 